\title{
Tacrolimus versus anti-tumor necrosis factor agents for steroid-refractory active ulcerative colitis based on the severity of endoscopic findings: a single-center, open-label cohort study
}

This article was published in the following Dove Press journal:

Clinical and Experimental Gastroenterology

26 September 2017

Number of times this article has been viewed

\section{Satohiro Matsumoto \\ Haruna Kawamura \\ Takeshi Nishikawa \\ Noriyoshi Sagihara \\ Hiroyuki Miyatani \\ Hirosato Mashima}

Department of Gastroenterology, Saitama Medical Center, Jichi Medical University, Saitama, Saitama, Japan
Correspondence: Satohiro Matsumoto Department of Gastroenterology, Saitama Medical Center, Jichi Medical University, I-847 Amanuma, Omiya, Saitama, Saitama 330-8503, Japan

$\mathrm{Tel}+814864721$ II

Fax +8I 486485188

Email s.w.himananon@ac.auone-net.jp
Background and aims: At Saitama Medical Center, for remission induction in active ulcerative colitis (UC) patients with endoscopic evidence of severe disease, we tend to preferentially use tacrolimus (TAC) over anti-tumor necrosis factor (TNF)- $\alpha$ agents. We conducted this study to evaluate the validity of our therapeutic strategies.

Patients and methods: This retrospective study was conducted in 52 steroid-refractory active UC patients with a Clinical Activity Index (CAI) score of $\geq 7$ who were receiving remission induction therapy with TAC or anti-TNF- $\alpha$ agents. The patients were divided into a TAC treatment group (TAC group, $\mathrm{n}=29$ ) and an anti-TNF- $\alpha$ agent treatment group (anti-TNF group, $\mathrm{n}=23$ ). The CAI, Ulcerative Colitis Endoscopic Index of Severity (UCEIS) and incidence of events (relapse, hospitalization and surgery) were retrospectively analyzed.

Results: At treatment initiation, the CAI score was 12.6 in the TAC group and 11.5 in the anti-TNF group $(P=0.09)$, while the corresponding values of the UCEIS were 6.5 and 5.1, respectively $(P=0.0035)$. The clinical remission rate at 12 weeks was $55 \%(65 \%$ when only the subgroup that received rapid induction therapy was included in the analysis) in the TAC group and $57 \%$ in the anti-TNF group, with no significant difference. The cumulative event-free rates at 1,6 and 12 months were $65.5 \%, 39.4 \%$, and $39.4 \%$, respectively, in the TAC group and $95.7 \%, 77.2 \%$ and $71.7 \%$, respectively, in the anti-TNF group $(P=0.0037)$.

Conclusion: Rapid induction therapy with TAC tended to be selected for active UC patients with endoscopic evidence of severe disease, and the present study supported the validity of this therapeutic approach. However, transition to the remission-maintenance phase was more favorable in the anti-TNF group.

Keywords: ulcerative colitis, tacrolimus, infliximab, adalimumab, steroid refractory

\section{Introduction}

Ulcerative colitis (UC) is a chronic inflammatory disease of the large intestine of unknown cause. In Japan, the number of patients with this disease is increasing annually, with the number of registered patients reaching 180,000 in the year 2014. Many patients with UC require remission induction therapy with steroids; however, in some cases, steroid withdrawal after successful remission induction is difficult, and in others, remission fails to be achieved. It is reported that among the patients with UC receiving systemic steroid therapy, $16 \%$ show resistance to steroids and $22 \%$ become dependent on steroids after 1 year of treatment. ${ }^{1}$ In patients who become dependent on or resistant to steroid therapy, tacrolimus (TAC) or anti-tumor necrosis factor (TNF)- $\alpha$ agents are 
used for remission induction therapy, and the efficacies of these medications have been assessed. ${ }^{2-6}$

Although TAC and anti-TNF- $\alpha$ agents are recognized as important therapeutic agents for $\mathrm{UC}$, there is currently no established means of distinguishing between these agents for use in remission induction therapy. At our hospital, we use TAC more frequently than anti-TNF agents for remission induction in patients with active refractory UC showing endoscopic evidence of relatively severe disease, such as mucosal shedding.

In this study, we attempted to assess the validity of our therapeutic strategy mentioned earlier, in terms of selecting between TAC and anti-TNF- $\alpha$ agents for remission induction therapy, as well as the outcomes and long-term prognosis of patients administered remission induction therapy with these agents.

\section{Patients and methods}

\section{Subjects}

This was a retrospective single center study at Saitama Medical Center. Of 495 patients with UC who had received inpatient or outpatient treatment at Saitama Medical Center between 2009 and 2015, 66 who had been treated with TAC or anti-TNF- $\alpha$ agents were selected, and their outcomes were assessed in October 2016. The patient inclusion criteria were as follows:
1) age $\geq 16$ years; 2) TAC or anti-TNF- $\alpha$ administered agents for remission induction therapy and 3) Clinical Activity Index (CAI) $\geq 7$ at the start of the remission induction therapy. The exclusion criteria were as follows: 1$)$ treatment with TAC or anti-TNF- $\alpha$ agents initiated at other hospitals; 2) CAI $\leq 6$ at the start of remission induction therapy; 3 ) history of colectomy and 4) referral back to the previous physicians after the start of remission induction therapy. Finally, 52 patients (34 men and 18 women; mean age at onset, $36 \pm 17$ years; mean disease duration, $4.5 \pm 4.6$ years) were included (Figure 1). Of these 52 patients, 29 had received TAC and 23 had received anti-TNF- $\alpha$ agents (including infliximab in 14 patients and adalimumab in nine patients).

UC was diagnosed on the basis of the characteristic endoscopic and biopsy findings, after excluding other inflammatory bowel disorders. Steroid treatment history included administration of steroidal agents orally, as enemas, or by injection in both the short term and the long term. Steroid dependence was defined as recurrence occurring during tapering of the steroid dose, and steroid resistance was defined as the absence of response to prednisolone administered at the dose of $1-1.5 \mathrm{mg} / \mathrm{kg} /$ day for $1-2$ weeks. Cytomegalovirus (CMV) infection was determined to be present when one of the following tests yielded positive results: serum CMV antigen test using horseradish peroxidase-labeled monoclonal

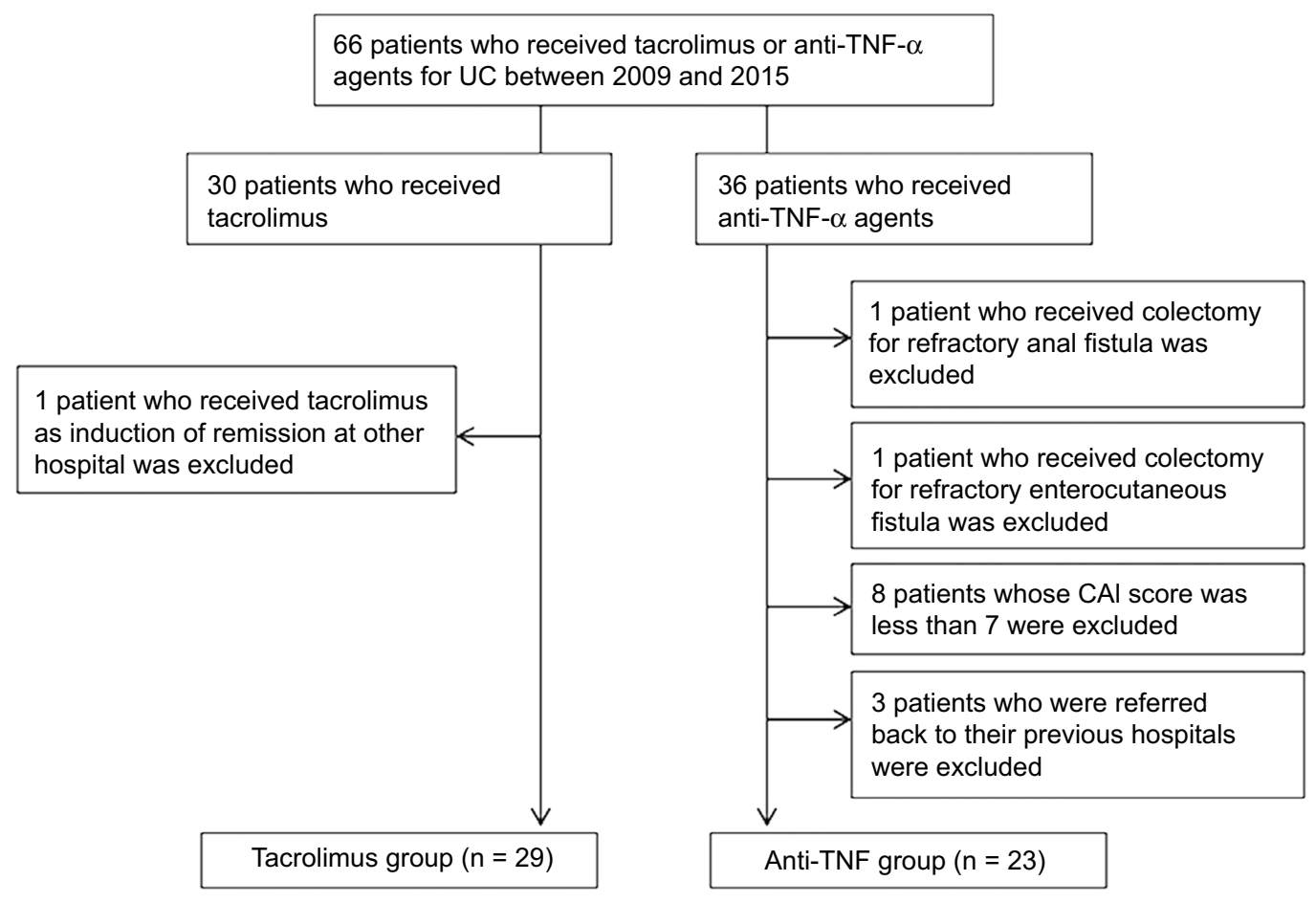

Figure I Flowchart of patient inclusion and exclusion.

Abbreviations: TNF, tumor necrosis factor; UC, ulcerative colitis; CAI, Clinical Activity Index. 
antibody (C7-HRP) or a cocktail of CMV monoclonal antibodies $(\mathrm{C} 10 / \mathrm{C} 11)$ and histopathological examination of colonic mucosal biopsy specimens for CMV infection.

\section{Treatment protocol}

Blood concentrations of TAC were measured with the Siemens EMIT 2000 measurement system at our hospital laboratory. Two induction protocols with TAC were available: the rapid induction protocol $(0.1 \mathrm{mg} / \mathrm{kg} /$ day $)$ and the standard induction protocol, in compliance with the instructions on the package insert $(0.05 \mathrm{mg} / \mathrm{kg} /$ day $)$. The doses of TAC were adjusted so as to achieve a target blood concentration of $10-15 \mathrm{ng} / \mathrm{mL}$ until week 2 and $5-10 \mathrm{ng} / \mathrm{mL}$ thereafter. Infliximab was administered at the dose of $5 \mathrm{mg} / \mathrm{kg}$ at weeks 0,2 and 6 , followed by a maintenance dose every 8 weeks. Adalimumab was administered at the dose of $160 \mathrm{mg}$ at week 0 and $80 \mathrm{mg}$ at week 2 , followed by $40 \mathrm{mg}$ every 2 weeks. The attending physicians selected one of these protocols, at their discretion, in all cases.

\section{Efficacy evaluation}

The patients were divided into two groups: those treated with TAC (TAC treatment group) and those treated with antiTNF- $\alpha$ agents (anti-TNF- $\alpha$ agent treatment group). Clinical symptom severity was quantified using the CAI developed by Lichtiger et al. ${ }^{7}$ The CAI is measured based on the following items: bowel movement frequency (scores $0-4$ ), presence/ absence of nocturnal diarrhea (scores $0-1$ ), presence/absence/ amount of blood in the stool (scores $0-3$ ), presence/absence of fecal incontinence (scores $0-1$ ), use/no use of antidiarrheal drugs (scores $0-1$ ), presence/absence/severity of abdominal pain (scores 0-3), general well-being (scores $0-5$ ) and the degree of abdominal tenderness (scores $0-3$ ). Clinical remission was defined as a $\mathrm{CAI}$ of $\leq 4$, and clinical response was defined as a CAI of $<10$ or a decrease in the CAI by 3 points after treatment initiation. Endoscopic findings were quantified using the Ulcerative Colitis Endoscopic Index of Severity (UCEIS) developed by Travis et al. ${ }^{8}$ The value of the UCEIS was determined based on the following three items: vascular pattern (scores 0-2), severity of bleeding (scores 0-3) and severity of erosion/ulceration (scores $0-3$ ). The sum of the scores for each endoscopic variable ranges from 0 to 8 . The scores for each patient were determined by assessment of the areas showing the most severe inflammation. Events were defined as additional therapy with prednisolone, hospitalization due to exacerbation of UC and colectomy.

The following parameters were retrospectively analyzed and assessed: 1) CAI at 0, 2, 4 and 12 weeks after the start of remission induction therapy; 2) clinical remission and clinical response rates at 12 weeks; 3) UCEIS value at the baseline and at 3-6 months after the start of remission induction therapy; 4) cumulative event-free rates and 5) cumulative colectomy rates. Adverse events were defined as any event that occurred during the administration of the drug.

\section{Ethics statement}

This study was conducted with the approval of the Etiological Study Ethical Review Board of Saitama Medical Center, Jichi Medical University (S17-002). Because we produced anonymized data for use in this study, it was deemed not necessary to obtain informed consent from the study subjects.

\section{Statistical analysis}

Data are expressed as mean \pm standard deviation or as percentages. The demographic characteristics of the study subjects were compared using Student's $t$-test and Fisher's exact test. The cumulative event-free rates were evaluated by the Kaplan-Meier method and compared using the log-rank test. All data analyses were performed with the StatView software (version 5.0; SAS Institute Inc., Cary, NC, USA). Differences at $P$-values of $<0.05$ were regarded as significant.

\section{Results}

The baseline characteristics of the study subjects are shown in Table 1. No significant differences in the sex ratio, diseasetype distribution, age at onset or disease duration were observed between the TAC and anti-TNF groups. The CAI score at treatment initiation was $12.7 \pm 2.6$ in the TAC group and $11.5 \pm 2.4$ in the anti-TNF group, tending to be higher in the former $(P=0.0957)$. The corresponding values of the UCEIS at treatment initiation were $6.4 \pm 1.3$ and $5.1 \pm 1.7$, respectively, being significantly higher in the TAC group $(P=0.0035)$. Moreover, the prevalence of CMV infection was $27.6 \%$ in the TAC group, which was also significantly higher than that of $4.3 \%$ in the anti-TNF group $(P=0.0336)$. In regard to concomitant drug use, immunomodulators had been used in $34.5 \%$ of patients of the TAC group and $69.6 \%$ of patients of the anti-TNF group; the rate of concomitant drug use was significantly higher in the latter $(P=0.0245)$. The anti-TNF group included a significantly higher number of patients with steroid dependence, whereas the TAC group included a significantly higher number of patients with steroid resistance. As for the blood test results, the serum C-reactive protein level was $5.5 \pm 4.8 \mathrm{mg} / \mathrm{dL}$ in the TAC group, which was significantly higher than the level of $1.7 \pm 2.0 \mathrm{mg} / \mathrm{dL}$ in the anti-TNF group $(P=0.0008)$; however, there were 
Table I Baseline characteristics

\begin{tabular}{|c|c|c|c|c|}
\hline Characteristic & Total $(n=52)$ & $\operatorname{TAC}(n=29)$ & Anti-TNF $(n=23)$ & $P$-value \\
\hline Male, n (\%) & $34(65.4)$ & $18(62.1)$ & $16(69.6)$ & 0.79 \\
\hline Age at onset (years), mean \pm standard deviation & $37 \pm 18(14-78)$ & $39 \pm 20(14-76)$ & $34 \pm 16(16-78)$ & 0.26 \\
\hline Extent at diagnosis, $\mathrm{n}(\%)$ & & & & 0.20 \\
\hline Total colitis & $39(75.0)$ & $24(82.8)$ & $15(65.2)$ & \\
\hline Left-sided colitis & $13(25.0)$ & $5(17.2)$ & $8(34.8)$ & \\
\hline Duration of UC (years), mean \pm standard deviation & $4.7 \pm 4.8(0-18.0)$ & $3.8 \pm 4.4(0-11.8)$ & $5.9 \pm 5.2(0.1-18.0)$ & 0.11 \\
\hline Current smoking, n (\%) & $10(19.2)$ & $3(10.3)$ & $7(30.4)$ & 0.08 \\
\hline \multicolumn{5}{|l|}{ Medical treatment, n (\%) } \\
\hline Mesalazine, salazosulfapyridine & $52(100)$ & $29(100)$ & $23(100)$ & 1.00 \\
\hline Immunomodulator & $26(50.0)$ & $10(34.5)$ & $16(69.6)$ & 0.0245 \\
\hline Prior anti-TNF agents & $3(4.8)$ & $2(6.9)$ & I (4.3) & 1.00 \\
\hline Prior cytapheresis & $31(59.6)$ & $19(62.5)$ & $12(52.2)$ & 0.39 \\
\hline Corticosteroid & $52(100)$ & $29(100)$ & $23(100)$ & 1.00 \\
\hline Steroid dependence & $32(61.5)$ & $13(44.8)$ & $19(82.6)$ & 0.0227 \\
\hline Steroid resistance & $20(38.5)$ & $16(55.2)$ & $4(17.4)$ & 0.0092 \\
\hline CMV infection, n (\%) & $9(17.3)$ & $8(27.6)$ & I (4.3) & 0.0336 \\
\hline \multicolumn{5}{|l|}{ Blood examination, mean \pm standard deviation } \\
\hline Hemoglobin $(g / L)$ & $10.9 \pm 2.2(6.8-15.8)$ & $10.5 \pm 2.1(7.0-15.3)$ & $11.4 \pm 2.3(6.8-15.8)$ & 0.16 \\
\hline Leukocyte count (10\%/L) & $9.7 \pm 4.4(4.0-28.8)$ & $10.6 \pm 5.0(4.7-28.8)$ & $8.5 \pm 3.3(4.0-17.0)$ & 0.09 \\
\hline Platelet count $\left(10^{4} / \mathrm{L}\right)$ & $45.2 \pm 14.4(14.7-80.3)$ & $48.0 \pm 15.2(14.7-80.3)$ & $41.6 \pm 12.8(26.0-77.0)$ & 0.11 \\
\hline $\mathrm{CRP}(\mathrm{mg} / \mathrm{dL})$ & $3.8 \pm 4.2(0.5-16.8)$ & $5.5 \pm 4.8(0.5-16.8)$ & $1.7 \pm 2.0(0.5-6.8)$ & 0.0008 \\
\hline $\begin{array}{l}\text { CAl at start of induction treatment, mean } \pm \text { standard } \\
\text { deviation }\end{array}$ & $12.2 \pm 2.6(7-12)$ & $12.7 \pm 2.6(7-19)$ & $11.5 \pm 2.4(7-16)$ & 0.09 \\
\hline $\begin{array}{l}\text { UCEIS at start of induction treatment, mean } \pm \text { standard } \\
\text { deviation }\end{array}$ & $5.8 \pm 1.6(1-8)$ & $6.4 \pm 1.3(4-8)$ & $5.1 \pm 1.7(1-8)$ & 0.0035 \\
\hline
\end{tabular}

Abbreviations: TAC, tacrolimus; TNF, tumor necrosis factor; UC, ulcerative colitis; CMV, cytomegalovirus; CRP, C-reactive protein; CAI, Clinical Activity Index; UCEIS, Ulcerative Colitis Endoscopic Index of Severity.

Table 2 Clinical outcomes

\begin{tabular}{|c|c|c|c|c|}
\hline Outcome & Total $(n=52)$ & TAC $(n=29)$ & Anti-TNF $(n=23)$ & $P$-value \\
\hline CAI at week 12 , mean \pm standard deviation & $4.1 \pm 3.0(0-10)$ & $3.1 \pm 2.7(0-10)$ & $4.9 \pm 3.0(1-10)$ & 0.05 \\
\hline Clinical remission rate at week $12(\%)$ & 55.8 & 55.1 & 56.5 & 1.00 \\
\hline Clinical response rate at week $12(\%)$ & 67.3 & 58.6 & 78.2 & 0.14 \\
\hline Hospitalization for UC exacerbation, $\mathrm{n}(\%)$ & $13(25.0)$ & $9(31.0)$ & $4(17.4)$ & 0.34 \\
\hline Colectomy, n (\%) & $15(28.8)$ & II (37.9) & $4(17.4)$ & 0.13 \\
\hline Adverse events, $\mathrm{n}(\%)$ & $7(13.5)$ & $7(24.1)$ & 0 & 0.0135 \\
\hline Complicating disease, $\mathrm{n}(\%)$ & $6(11.5)$ & $5(17.2)$ & $\mathrm{I}(4.3)$ & 0.21 \\
\hline $\mathrm{UC}$ related death, $\mathrm{n}(\%)$ & I (I.9) & I (3.4) & 0 & 1.00 \\
\hline Observation period (years), mean \pm standard deviation & $3.8 \pm 1.9(0.1-7.3)$ & $4.1 \pm 1.9(0.1-7.3)$ & $3.5 \pm 1.8(1.2-6.4)$ & 0.30 \\
\hline
\end{tabular}

Abbreviations: TAC, tacrolimus; TNF, tumor necrosis factor; CAI, Clinical Activity Index; UC, ulcerative colitis.

no significant differences in any of the other parameters examined. The mean TAC concentrations $(\mathrm{ng} / \mathrm{mL})$ on days $2-3,7,14$ and 28 were $8.7 \pm 7.6,10.5 \pm 4.0,12.3 \pm 4.1$ and $9.1 \pm 4.7$, respectively; thus, the target blood concentrations were achieved and maintained.

The clinical outcomes in each group are shown in Table 2. The CAI scores at $0,2,4$ and 12 weeks after the start of remission induction therapy were $12.7 \pm 2.6,6.1 \pm 3.1$, $4.6 \pm 3.3$ and $3.5 \pm 3.1$, respectively, in the TAC group, and the corresponding scores in the anti-TNF group were $11.5 \pm 2.4,6.3 \pm 3.2,5.0 \pm 3.3$ and $5.2 \pm 3.0$, respectively, indicating statistically significant decreases in both groups
( $P<0.0001$, both; Figure 2A). The corresponding values of the UCEIS at the baseline and at 3-6 months after the start of remission induction therapy were $6.4 \pm 1.3$ and $4.7 \pm 2.0$ $(P=0.0017)$ and $5.1 \pm 1.7$ and $2.9 \pm 1.8$, respectively $(P=0.0011)$, indicating statistically significant decreases in both groups (Figure 2B). The clinical remission rate at 12 weeks after the start of remission induction therapy was $55 \%$ in the TAC group and 57\% in the anti-TNF group. However, when only the data of patients who had received rapid induction therapy in the TAC group were included in the analysis, the clinical remission rate at 12 weeks was $65 \%$. The clinical response rates were $59 \%$ and $78 \%$ in the TAC 
and anti-TNF groups, respectively, showing no significant difference between the two groups. Seven of eight patients of the TAC group and one patient of the anti-TNF group with CMV infection received ganciclovir. We also performed a subgroup analysis by dividing the patients of the anti-TNF group into infliximab and adalimumab groups. The CAI scores at $0,2,4$ and 12 weeks after the start of remission induction therapy were $11.7 \pm 2.6,5.5 \pm 3.4,4.1 \pm 3.2$ and
$4.6 \pm 3.1$ in the infliximab group and $11.2 \pm 2.2,7.5 \pm 2.5$, $6.2 \pm 3.1$ and $5.4 \pm 2.9$ in the adalimumab group, respectively, indicating statistically significant decreases in the scores of both groups $(P<0.0001$, both) and a nonsignificant difference between both groups (Figure 3A). The values of the UCEIS at the baseline and at 3-6 months after the start of remission induction therapy were $5.0 \pm 1.7$ and $2.7 \pm 1.6$ in the infliximab group $(P=0.0127)$ and $5.3 \pm 1.9$ and $3.3 \pm 2.2$
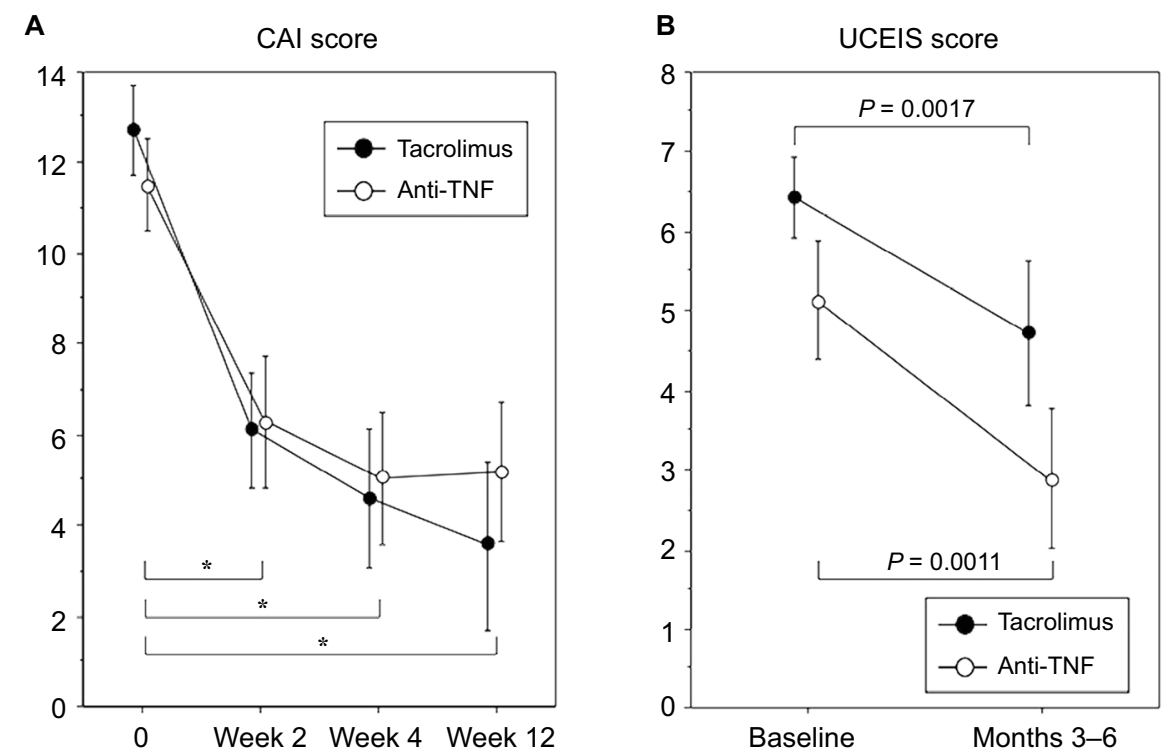

Figure 2 Transitional change of CAI and UCEIS.

Notes: (A) CAl of Lichtiger score at weeks 0, 2, 4 and 12 after the start of induction therapy. (B) UCEIS score at the baseline and months 3-6 in the TAC group and antiTNF group. *significant difference $(P<0.0001)$.

Abbreviations: CAI, Clinical Activity Index; UCEIS, Ulcerative Colitis Endoscopic Index of Severity; TAC, tacrolimus; TNF, tumor necrosis factor.
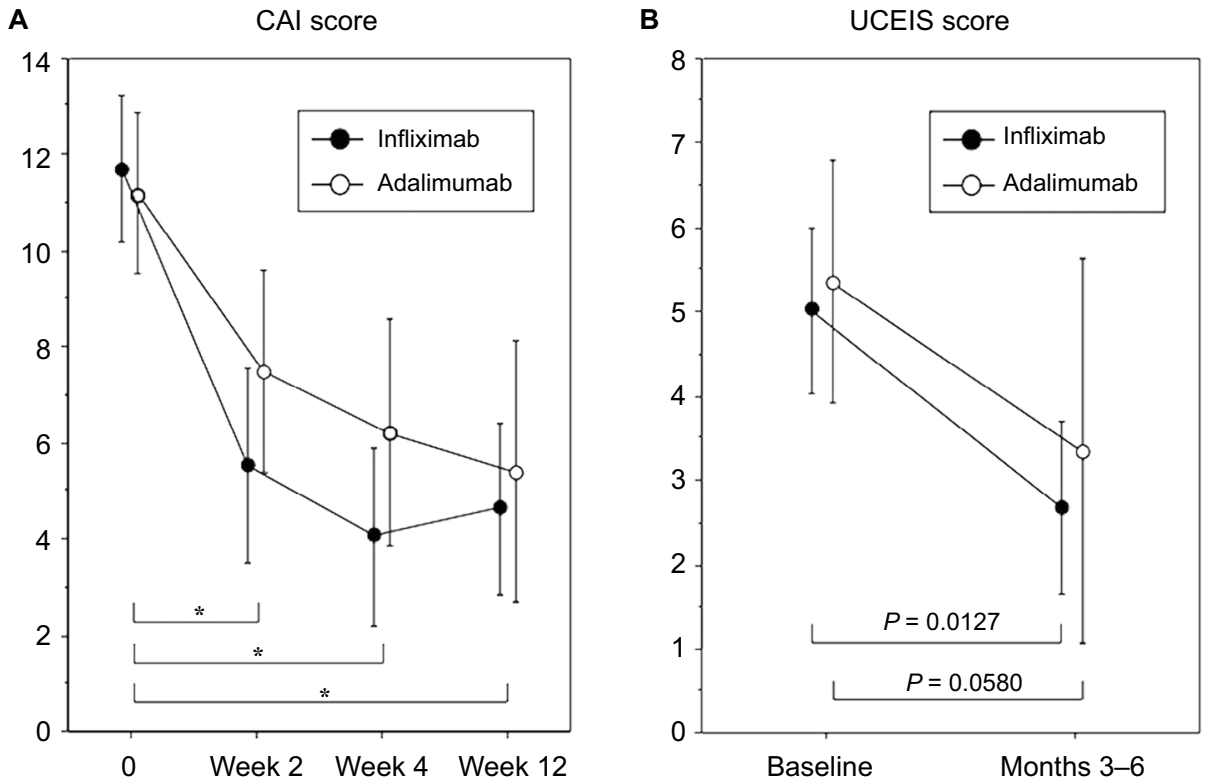

Figure 3 Transitional change of $\mathrm{CAI}$ and UCEIS.

Notes: (A) CAI of Lichtiger score at weeks 0, 2, 4 and 12 after the start of induction therapy. (B) UCEIS score at the baseline and months 3-6 in the infliximab group and adalimumab group. *significant difference $(P<0.000 \mathrm{I})$.

Abbreviations: CAI, Clinical Activity Index; UCEIS, Ulcerative Colitis Endoscopic Index of Severity. 
in the adalimumab group $(P=0.0580)$, respectively, indicating significant decreases only in the infliximab group (Figure 3B). The clinical remission rate at 12 weeks after the start of remission induction therapy was $57 \%$ in the infliximab group and $56 \%$ in the adalimumab group.

The cumulative event-free rates at 1,6 and 12 months after treatment initiation were $65.5 \%, 39.4 \%$, and $39.4 \%$, respectively, in the TAC group and $95.7 \%, 77.2 \%$ and $71.7 \%$, respectively, in the anti-TNF group, the rates being significantly higher in the latter group $(P=0.0037$; Figure 4$)$. During the study period, the percentage of patients who needed hospitalization because of UC relapse was $31.0 \%$ in the TAC group and $17.4 \%$ in the anti-TNF group. Colectomy was performed in $37.9 \%$ of the patients of the TAC group and $17.4 \%$ of the patients of the anti-TNF group; thus, there was no significant difference in the percentage of patients who underwent colectomy between the two groups (Table 2). The cumulative colectomy rates at 1, 6 and 12 months after treatment initiation were $24.5 \%, 28.2 \%$ and $37.8 \%$ in the TAC group and $0 \%, 13.6 \%$ and $18.7 \%$ in the anti-TNF group $(P=0.0531$; Figure 5).

Flow charts of the clinical course in the two groups are shown in Figure 6. In all the 17 patients of the TAC group who showed a clinical response, the treatment agent was switched from TAC to thiopurine. Maintenance therapy was continued in $10(58.8 \%)$ of these patients, whereas the remaining seven patients experienced relapse during the follow-up period. In five of the seven patients with relapse, the TAC had been withdrawn after 3 months of treatment. On the other hand, maintenance therapy with anti-TNF- $\alpha$ agents could be continued without any changes in $13(72.2 \%)$ of the patients

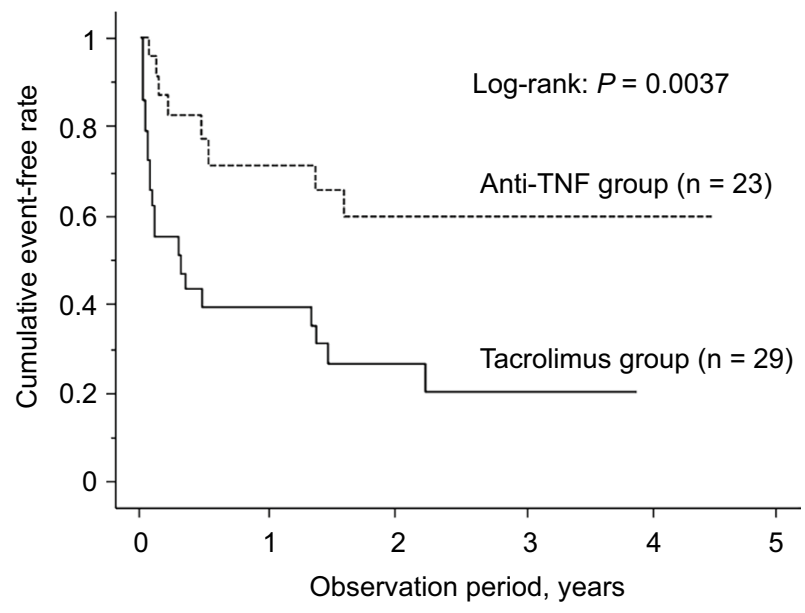

Figure 4 Kaplan-Meier analysis of the cumulative event-free rate. Note: Events were defined as additional therapy with prednisolone, hospitalization due to exacerbation of UC and colectomy. Abbreviations: UC, ulcerative colitis; TNF, tumor necrosis factor. of the anti-TNF group who showed a clinical response; the remaining five patients experienced relapse.

Adverse reactions were observed in $24.1 \%$ (7/29) of patients of the TAC group, including hepatic dysfunction in two patients, renal dysfunction in two patients, hypomagnesemia in two patients and tremor in one patient. No adverse reactions were encountered in any patient of the anti-TNF group $(P=0.0135)$. Complications were observed in $17.2 \%$ $(5 / 29)$ of patients of the TAC group, including new CMV infection in two patients, central venous catheter infection in two patients and phlegmon in the left forearm in one patient. In the anti-TNF group, central venous catheter infection was observed in $4.3 \%$ (1/23) of patients. Although very few patients had serious clinical courses, one patient of the TAC group died of complications of UC. This patient was a 75-year-old woman who developed toxic megacolon during the follow-up period and underwent total colectomy. The postoperative course was unfavorable. She developed Pneumocystis pneumonia 1 month after the surgery and died of hemorrhagic shock 4 months after the surgery (Table 2).

\section{Discussion}

In Japan, National Health Insurance reimbursement approvals for use of TAC, infliximab, adalimumab and infliximab biosimilar in the treatment of refractory UC were obtained in 2009, 2010, 2013 and 2014, respectively. Although TAC and anti-TNF- $\alpha$ agents are recognized as important therapeutic agents for refractory $\mathrm{UC}$, there are very few reports focusing on distinguishing between these two drug classes for remission induction therapy in UC patients. Evidence to support the use of one over the other drug class is thus scarce. At our hospital, we use TAC more often than anti-TNF- $\alpha$ agents

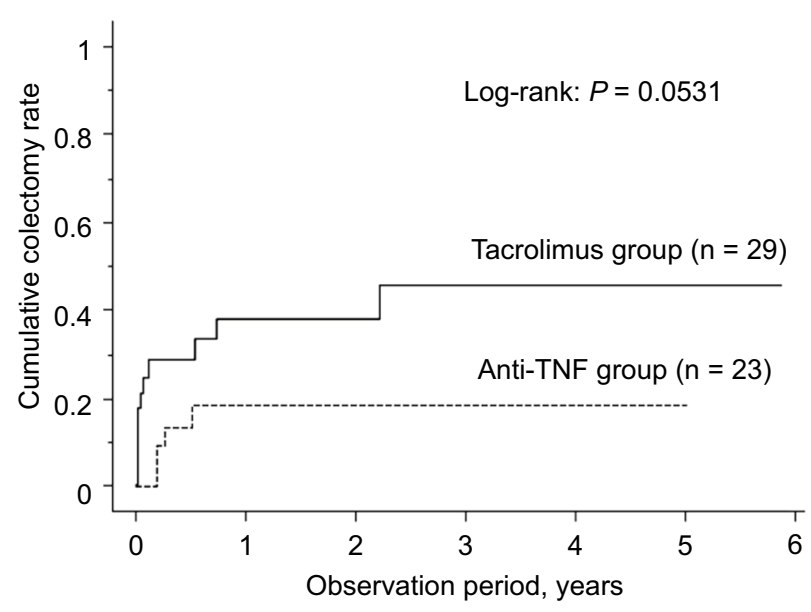

Figure 5 Kaplan-Meier analysis of the cumulative colectomy rate. Abbreviations: TNF, tumor necrosis factor. 

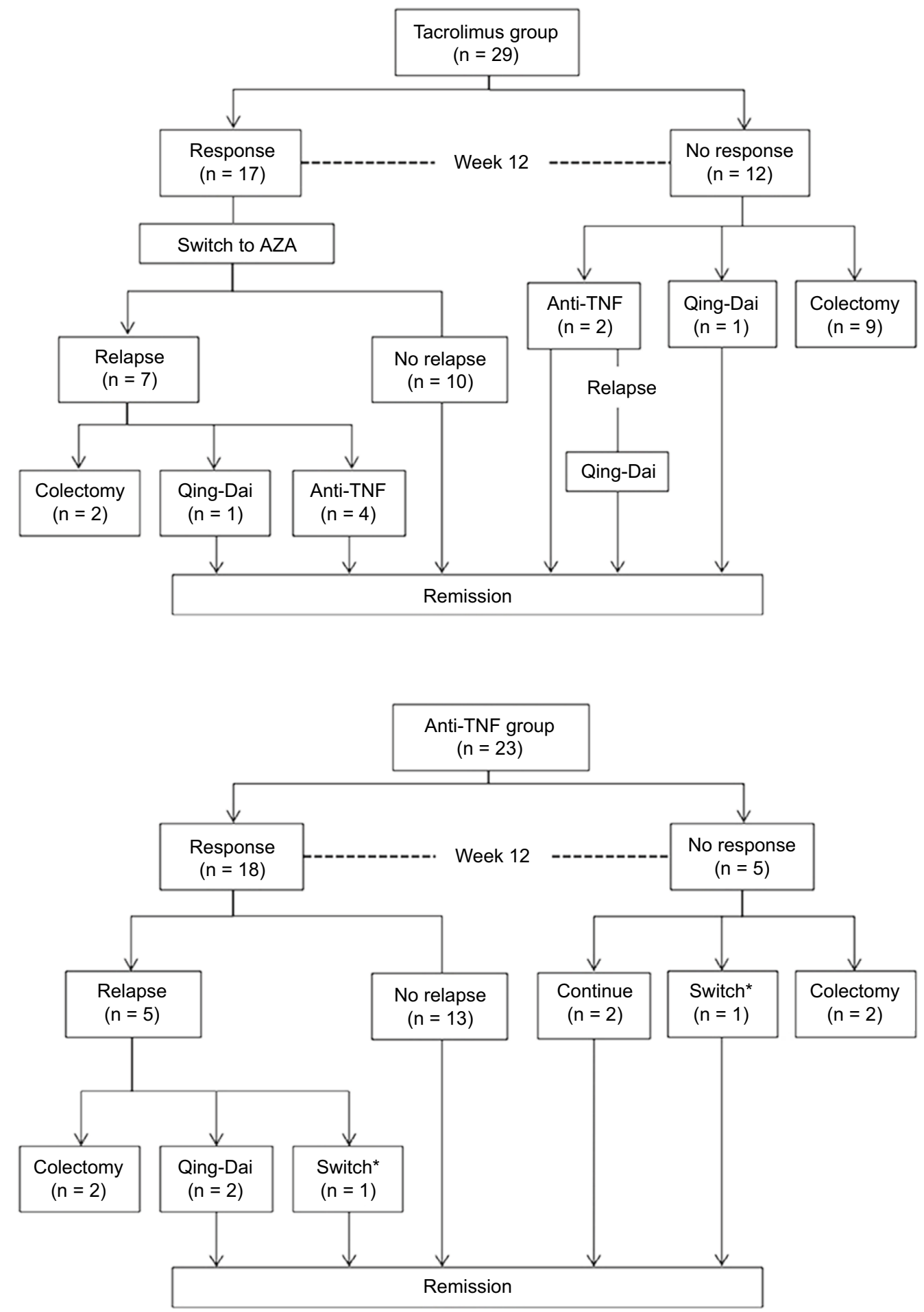

Figure 6 Flowchart of outcomes of UC patients treated with TAC or anti-TNF agents.

Notes: Qing-Dai is a Chinese herbal medicine and has used to treat UC patients. ${ }^{24}$ Switch*, switch to another anti-TNF agents.

Abbreviations: UC, ulcerative colitis; TAC, tacrolimus; TNF, tumor necrosis factor; AZA, azathioprine.

for induction of remission in patients with refractory $\mathrm{UC}$ showing endoscopic evidence of relatively severe UC, such as mucosal shedding. In the present retrospective study, we attempted to validate our strategy of preferential use of TAC for the treatment of severe UC.
TAC has a potent immunosuppressive effect. Even oral preparations of this drug are well absorbed from the intestinal tract, which facilitates achievement of a constant blood concentration. Ogata et $\mathrm{al}^{2}$ demonstrated in a multicenter, double-blind study that administration of oral TAC 
to obtain a blood trough level of $10-15 \mathrm{ng} / \mathrm{mL}$ is an effective technique for remission induction therapy in patients with steroid-resistant UC. The drug has since been used at a dosage schedule that allows the above-described blood concentrations to be achieved as soon as possible after treatment initiation. Meanwhile, in regard to the clinical efficacy and safety of infliximab and adalimumab for treating UC, the Active Ulcerative Colitis Trial (ACT) 1 for infliximab and the Ulcerative Colitis Long-term Remission and Maintenance with Adalimumab (ULTRA) 2 trial for adalimumab demonstrated that both drugs were significantly more effective than placebo in improving the clinical symptoms within a short period and in inducing remission. ${ }^{6,9}$ In a retrospective study conducted by Yamamoto et $\mathrm{al}^{10}$ to compare anti-TNF- $\alpha$ agents with TAC in patients with steroid-refractory UC, no significant differences in efficacy were observed during the first 12 weeks of treatment. Endo et $\mathrm{al}^{11}$ also reported the absence of any significant differences in the colectomy-free, clinical remission and clinical response rates at 2 months after the start of treatment between patients treated with anti-TNF- $\alpha$ agents and those treated with TAC. In the present study, the clinical remission rate at 12 weeks after the start of remission induction therapy was $55 \%$ in the TAC group and $57 \%$ in the anti-TNF group. Although the rate was $65 \%$ when only patients receiving rapid induction therapy in the TAC group were included in the analysis, this rate was not significantly different from that in the anti-TNF group. However, the UCEIS and prevalence of CMV infection at the start of remission induction therapy were significantly higher in the TAC group. Furthermore, given that TAC was administered to patients with endoscopic evidence of relatively severe disease, we assume that our strategy for selecting TAC over anti-TNF agents for remission induction is valid in terms of distinguishing between these two types of drugs as to their use. In all patients treated with ganciclovir, prompt conversion to a CMV infection-negative state was obtained; thus, $\mathrm{CMV}$ infection did not have any profound influence on the clinical outcomes.

The major advantage of anti-TNF- $\alpha$ antibodies is that they can also be used for maintenance therapy after remission induction. The ACT 1 on infliximab and the ULTRA 2 trial on adalimumab demonstrated that both drugs provide sustained clinical symptom control and also yield significantly better remission-maintenance rates than placebo. ${ }^{6,9}$ Furthermore, $60 \%$ of the patients in whom clinical remission and mucosal healing were achieved remained in remission for 4 years. ${ }^{12}$ On the other hand, long-term remission-maintenance therapy with TAC is currently not approved for National Health
Insurance reimbursement in Japan. In a study conducted by Yamamoto et $\mathrm{al}^{9}$ in patients with refractory UC who received long-term therapy with TAC for a mean duration of 11 months, the operation-free rate at 65 months was favorable at $62.3 \%$. In another study comparing anti-TNF- $\alpha$ agents with TAC for patients with steroid-refractory UC, Yamamoto et $\mathrm{al}^{10}$ reported that the majority of responders in the biologics group remained in remission at 52 weeks, whereas $16 \%$ of patients in the TAC group experienced relapse after treatment switch to azathioprine. Moreover, Endo et $\mathrm{al}^{11}$ reported that the long-term relapse rate was significantly lower in patients treated with anti-TNF- $\alpha$ agents than in those treated with TAC. The present study also showed that UC relapsed more frequently in patients of the TAC group in whom the drug was switched to thiopurine, while endoscopic remission had not yet been achieved. Thus, the timing of transition to treatment with immunomodulatory drugs is important. It has also been reported that the relapse rate is significantly lower in patients with a UCEIS of $\leq 1$ than in those with a UCEIS of $\geq 2^{13,14}$ and that TAC cannot be expected to be effective in repeated attempts to achieve remission induction. ${ }^{15}$ Thus, it seems preferable to switch from TAC to another treatment agent once endoscopic mucosal healing has been achieved. We consider it preferable to switch to maintenance therapy when endoscopy reveals a decrease in the Mayo Endoscopic Score to $\leq 1$ overall or $\leq 2$ for only local lesions. In the present study, TAC was administered for a relatively long period, i.e., $\geq 3$ months, in $41 \%$ of patients of the TAC group.

The reported adverse reactions to anti-TNF- $\alpha$ agents include susceptibility to infection, particularly tuberculosis, reactivation of hepatitis $B$ virus and development of nonmelanoma skin cancer and lymphoma. ${ }^{16-20}$ Screening for tuberculosis before administration of these agents is thus essential. ${ }^{21}$ A recent analysis of pooled data revealed that combined use of anti-TNF- $\alpha$ antibody agents and immunomodulators reduces the risk of infection at 1 year after the start of treatment, while the use of corticosteroids increases the risk of infection at 120 days after treatment. ${ }^{22}$ On the other hand, TAC has a narrow therapeutic window. If blood concentrations are high, serious adverse reactions, such as renal dysfunction, can occur. Great interindividual and intraindividual fluctuations are observed in the biokinetics. Thus, when TAC is used, it is essential to prepare an individualized administration plan based on monitoring of the blood concentrations of this drug. Because the incidence of adverse reactions is higher when the blood trough level is $\geq 10 \mathrm{ng} / \mathrm{mL},{ }^{23}$ attention should be paid to excessive increases in the concentrations and the occurrence of adverse reactions during the early induction phase 
of TAC therapy with a target concentration of $\geq 10 \mathrm{ng} / \mathrm{mL}$. In the present study, the incidence of both adverse reactions and complications tended to be higher in the TAC group than in the anti-TNF group, although the differences did not reach statistical significance. When TAC is used, concomitant administration of sulfamethoxazole-trimethoprim may need to be considered to prevent Pneumocystis pneumonia, depending on the clinical situation.

The present study has the following limitations: It was a single-center, small-scale retrospective cohort study. Therefore, there was a selection bias. TAC was discontinued within 3 months of the start of treatment in the first six cases of the TAC group in this study, because TAC administration for $>3$ months was not yet approved in Japan. Therefore, there was an admixture of patients who received TAC for $<3$ and $>3$ months in the TAC group in this study. While two induction protocols with TAC are available, namely, the rapid induction protocol and standard induction protocol in compliance with the package insert instructions, choosing between these two protocols was left to the discretion of the attending physicians in this study. Furthermore, the anti-TNF group consisted of a mixture of patients who had received infliximab and those who had received adalimumab. As mentioned earlier, in Japan, infliximab and adalimumab received approval for use as standard therapy for UC at different time points. Therefore, the mean observation period in the infliximab group was significantly longer than that in the adalimumab group (infliximab, $4.7 \pm 1.4$ years vs. adalimumab, $1.7 \pm 0.5$ years; $P<0.0001)$. The timing of endoscopy before and after the start of remission induction therapy was inconsistent among the patients.

\section{Conclusion}

Under circumstances that necessitate consideration of surgery, such as in active UC patients with endoscopic evidence of severe disease, administration of TAC by the rapid induction protocol appears to be a valid therapeutic option. Moreover, use of anti-TNF- $\alpha$ agents, which are effective for maintaining remission, also appears to be an effective therapeutic option for patients with active UC.

\section{Author contributions}

All authors contributed toward data analysis, drafting and revising the paper and agree to be accountable for all aspects of the work.

\section{Disclosure}

The authors report no conflicts of interest in this work.

\section{References}

1. Faubion WA Jr, Loftus EV Jr, Harmsen WS, Zinsmeister AR, Sandborn WJ. The natural history of corticosteroid therapy for inflammatory bowel disease: a population-based study. Gastroenterology. 2001;121(2):255-260.

2. Ogata $\mathrm{H}$, Matsui $\mathrm{T}$, Nakamura $\mathrm{M}$, et al. A randomised dose finding study of oral tacrolimus (FK506) therapy in refractory ulcerative colitis. Gut. 2006;55(9):1255-1262.

3. Baumgart DC, Pintoffl JP, Sturm A, Wiedenmann B, Dignass AU. Tacrolimus is safe and effective in patients with severe steroid-refractory or steroid-dependent inflammatory bowel disease--a long-term follow-up. Am J Gastroenterol. 2006;101(5):1048-1056.

4. Rutgeerts P, Sandborn WJ, Feagan BG, et al. Infliximab for induction and maintenance therapy for ulcerative colitis. $N$ Engl $J$ Med. 2005;353(23):2462-2476.

5. Järnerot G, Hertervig E, Friis-Liby I, et al. Infliximab as rescue therapy in severe to moderately severe ulcerative colitis: a randomized, placebo-controlled study. Gastroenterology. 2005;128(7): 1805-1811.

6. Sandborn WJ, van Assche G, Reinisch W, et al. Adalimumab induces and maintains clinical remission in patients with moderate-to-severe ulcerative colitis. Gastroenterology. 2012;142(2):257-265.

7. Lichtiger S, Present DH, Kornbluth A, et al. Cyclosporine in severe ulcerative colitis refractory to steroid therapy. N Engl J Med. 1994;330(26): 1841-1845.

8. Travis SP, Schnell D, Krzeski P, et al. Reliability and initial validation of the ulcerative colitis endoscopic index of severity. Gastroenterology. 2013;145(5):987-995.

9. Yamamoto S, Nakase H, Mikami S, et al. Long-term effect of tacrolimus therapy in patients with refractory ulcerative colitis. Aliment Pharmacol Ther. 2008;28(5):589-597.

10. Yamamoto T, Shimoyama T, Umegae S, Matsumoto K. Tacrolimus vs. anti-tumour necrosis factor agents for moderately to severely active ulcerative colitis: a retrospective observational study. Aliment Pharmacol Ther. 2016;43(8):705-716.

11. Endo K, Onodera M, Shiga H, et al. A comparison of short- and longterm therapeutic outcomes of infliximab- versus tacrolimus-based strategies for steroid-refractory ulcerative colitis. Gastroenterol Res Pract. 2016;2016:3162595.

12. Colombel JF, Sandborn WJ, Ghosh S, et al. Four-year maintenance treatment with adalimumab in patients with moderately to severely active ulcerative colitis: data from ULTRA 1, 2, and 3. Am J Gastroenterol. 2014;109(11):1771-1780.

13. Arai M, Naganuma M, Sugimoto $\mathrm{S}$, et al. The ulcerative colitis endoscopic index of severity is useful to predict medium- to long-term prognosis in ulcerative colitis patients with clinical remission. J Crohns Colitis. 2016;10(11):1303-1309.

14. Ikeya K, Sugimoto K, Kawasaki S, et al. Tacrolimus for remission induction in ulcerative colitis: mayo endoscopic subscore 0 and 1 predict long-term prognosis. Dig Liver Dis. 2015;47(5): $365-371$.

15. Hiraoka S, Kato J, Suzuki H, Yamamoto K. Readministration of calcineurin inhibitors for ulcerative colitis. Ann Pharmacother. 2012;46(10):1315-1321.

16. Bonovas S, Fiorino G, Allocca M, et al. Biologic therapies and risk of infection and malignancy in patients with inflammatory bowel disease: a systematic review and network meta-analysis. Clin Gastroenterol Hepatol. 2016;14(10):1385-1397.

17. Keane J, Gershon S, Wise RP, et al. Tuberculosis associated with infliximab, a tumor necrosis factor alpha-neutralizing agent. $N$ Engl $J$ Med. 2001;345(15):1098-1104.

18. Esteve M, Saro C, González-Huix F, Suarez F, Forné M, Viver JM. Chronic hepatitis B reactivation following infliximab therapy in Crohn's disease patients: need for primary prophylaxis. Gut. 2004;53(9): 1363-1365. 
19. Long MD, Martin CF, Pipkin CA, Herfarth HH, Sandler RS, Kappelman MD. Risk of melanoma and nonmelanoma skin cancer among patients with inflammatory bowel disease. Gastroenterology. 2012;143(2):390-399.

20. Khan N, Abbas AM, Lichtenstein GR, Loftus EV Jr, Bazzano LA. Risk of lymphoma in patients with ulcerative colitis treated with thiopurines: a nationwide retrospective cohort study. Gastroenterology. 2013;145(5):1007-1015.

21. Abitbol Y, Laharie D, Cosnes J, et al. Negative screening does not rule out the risk of tuberculosis in patients with inflammatory bowel disease undergoing anti-TNF treatment: a descriptive study on the GETAID cohort. J Crohns Colitis. 2016;10(10):1179-1185.
22. Osterman MT, Sandborn WJ, Colombel JF, et al. Crohn's disease activity and concomitant immunosuppressants affect the risk of serious and opportunistic infections in patients treated with adalimumab. $A m J$ Gastroenterol. 2016;111(12):1806-1815.

23. Opelz G. Effect of immunosuppressive therapy on graft half-life projections. The collaborative Transplant Study. Transplant Proc. 1999;31(7): 31-33.

24. Sugimoto S, Naganuma M, Kiyohara H, et al. Clinical efficacy and safety of oral Qing-Dai in patients with ulcerative colitis: a single-center open-label prospective study. Digestion. 2016;93(3):193-201.
Clinical and Experimental Gastroenterology

\section{Publish your work in this journal}

Clinical and Experimental Gastroenterology is an international, peerreviewed, open access, online journal publishing original research, reports, editorials, reviews and commentaries on all aspects of gastroenterology in the clinic and laboratory. This journal is included on PubMed. The manuscript management system is completely online
Dovepress

and includes a very quick and fair peer-review system, which is all easy to use. Visit http://www.dovepress.com/testimonials.php to read real quotes from published authors. 